\title{
BMC Pregnancy and Childbirth reviewer acknowledgement 2015
}

Nawsheen Boodhun

\section{Contributing reviewers}

The editors of BMC Pregnancy and Childbirth would like to thank all our reviewers who have contributed to the journal in Volume 15 (2015).

Annika Ahman
Sweden

Clara Aarts

Sweden

Paula Abate

Argentina

Parvin Abedi

Iran

Abdulai Abubakari

Germany

Ganesh Acharya

Norway

Princess Ruhama Acheampong

Ghana

Princess Acheampong

Ghana

Mulat Adefris

Ethiopia

Oyelola Adegboye

Belgium

Oluyinka Adejumo

USA

Visseho Adjiwanou

South Africa

Annsofie Adolfsson

Sweden
Schadrac Agbla

Benin

Gabriel Agboado

UK

Kingsley Agho

Australia

Eleonora Agricola

Italy

Faruk Ahmed

Australia

Azza Ahmed

USA

Saifuddin Ahmed

USA

Ashraful (Neeloy) Alam

Australia

Yueping Alex Wang

Australia

Nagi Ali

Sudan

Imran Ali Khan

Saudi Arabia

Hanan Al-Kadri

Saudi Arabia

Emma Allanson

Australia
Rebecca Allen

UK

Charles Ameh

UK

Amanda Ampt

Australia

Todd Anderson

Canada

Ewa Andersson

Sweden

Prabha Andraweera

Sri Lanka

Aisha Andrewin

Belize

Rosita Aniuliene

Lithuania

Gifty Dufie Antwi

Ghana

Olatunde Aremu

UK

Nigel Armfield

Australia

Raul Artal

USA

Umesh Raj Aryal

Nepal 
Nina Asplin

Sweden

Nega Assefa

Ethiopia

Roger Ayimbillah Atinga

Ghana

Lou Atkinson

UK

Carolyn Audet

USA

Dagfinn Aune

UK

Marlein Ausems

Netherlands

Pia Axemo

Sweden

Jennifer Ayton

Australia

Hassan Ba'Aqeel

Saudi Arabia

Nadia Badawi

Australia

Rashmi Bagga

India

Frank Baiden

Ghana

Emily Bain

Australia

Kathleen Baird

Australia

Martha Baird

USA

Marian Bakker

Netherlands

Marie-Clare Balaam

UK

Daynia E Ballot

South Africa

Julius Bamidele

Nigeria

Sushanta Banerjee

India

Aduragbemi Banke-Thomas

UK
Kesha Baptiste-Roberts

USA

Carol Barber

New Zealand

Flora Maria Barbosa Da Silva

Brazil

Kelli Barbour

USA

Lesley Barclay

Australia

Mary Barker

UK

Cheryl Barnabe

Canada

Margaret Barnes

Australia

Vendula Bartáková

Czech Republic

Subit Barua

USA

Hyam Bashour

Syrian Arab Republic

Stuart Basten

UK

Brian Bateman

USA

Sara Bayes

Australia

Hamideh Bayrampour

Canada

Alessandra Bazzano

USA

Arjun Bedi

Netherlands

Cecily Begley

Ireland

Loubna Belaid

Canada

Candice Belanoff

USA

Jacqueline Bell

UK

Katrien Benhalima

Belgium
Janie Benson

USA

Jason Bentley

Australia

Karen Benzies

Canada

Anick Berard

Canada

Marie Berg

Sweden

Howard Berger

Canada

Annette Bernloehr

Germany

Kerstin Berntorp

Sweden

Fabrizio Bert

Italy

Prem Bhandari

USA

Sohinee Bhattacharya

UK

Sanghita Bhattacharyya

India

Agnieszka Bianek-Bodzak

Poland

Jean Joel Bigna

Cameroon

Lorena Binfa

Chile

Mary Anne Biro

Australia

Uta Bischof

Germany

Concepción Blasco-Ros

Spain

Janusz Blaszczyk

Poland

Joan Bloch

USA

Karen Block

Australia

Marie Blomberg

Sweden 
Machteld Boel

Netherlands

Nansi Boghossian

USA

Meghan Bohren

USA

Carolyn Bolton Moore

Zambia

Paula Bolton-Maggs

UK

Igna Bonfrer

Netherlands

Oleg Borisenko

Sweden

Renata Bortolus

Italy

Sabine Bousleiman

USA

Angela Bowen

Canada

Jennifer Bowen

Australia

Marek Brabec

Czech Republic

Susan Bradley

UK

Vivienne Brady

Ireland

Olivier Braissant

Switzerland

Stephan Brenner

Germany

Alexandra Brentani

Brazil

Paula Brentlinger

USA

Christian Breymann

Switzerland

Lynne Briggs

Australia

Wendy Brodribb

Australia

Carinne Brody

USA
Gabriella Broms

Sweden

Louise Brough

New Zealand

Tim Bruckner

USA

Ashley Bryce

UK

Luc Budé

Netherlands

Pierre Buekens

USA

Wilma Buffolano

Italy

Cynthia Bulik

USA

John Burgess

Australia

Stefanie Burghaus

Germany

Lisa Butler

USA

Alexander Butwick

USA

Abbey Byrne

Australia

Robert Cabrera

USA

Karin Cadwell

USA

Joan Cameron

UK

Le Ray Camille

France

Sandra Campbell

Australia

Deborah Campbell

USA

Simona Cardaropoli

Italy

Mary Carolan-Olah

Australia

Scala Carolina

UK
Andrea Cassidy-Bushrow

USA

Arnaud Cénac

France

Luisa Cescutti-Butler

UK

Catherine Chamberlain

Australia

Yiong Huak Chan

Singapore

Edwin Chandraharan

UK

Yao-Lung Chang

Taiwan

Jen Jen Chang

USA

Anne Chantry

France

Katie Chaput

Canada

Madleen Chassang

France

Sarika Chaturvedi

India

Sanjukta Chaudhuri

USA

Shalini Chawla

UK

Dong-Bao Chen

USA

Brian Chen

USA

Kate Cheney

Australia

Andreas Chiabi

Cameroon

Matthew Chico

UK

Jobiba Chinkhumba

Malawi

Joanne Chiwaula

Ghana

Bishnu Choulagai

Nepal 
Mayora Chrispus

Uganda

Jones Chrissie

UK

Tereza Cindrova-Davies

UK

Ernestina Coast

UK

Judy Slome Cohain

Israel

Priscilla Coleman

USA

Katherine Collins

UK

Yvette Conley

USA

Cynthia D Connelly

USA

Amanda Cooklin

Australia

Robert Coombs

UK

Diane Cooper

South Africa

Enoka Corea

Sri Lanka

Denise Cote-Arsenault

USA

Kim Cox

USA

Debra Creedy

Australia

Ellen Cromley

USA

Coleen K. Cunningham

USA

Kenda Cunningham

USA

Rachel Cutting

UK

Allan Cyna

Australia

Fabricio Da Silva Costa

Australia
Rea Daellenbach

New Zealand

Darie Daemers

Netherlands

Rada Dagher

USA

Hannah Dahlen

Australia

Guoli Dai

USA

Philip Dalinjong

Australia

Anne Kjersti Daltveit

Norway

Deirdre Daly

Ireland

Per Damkier

Denmark

Kay Daniels

USA

Francesco D'Antonio

Italy

Edward T. Dassah

Ghana

Margie Davenport

Canada

Natalie Davidson

Australia

Miranda Davies-Tuck

Australia

Deborah Davis

Australia

Deborah Davis

USA

Luc De Bernis

Ethiopia

Caroline De Costa

Australia

Susie De Jersey

Australia

Ank De Jonge

Netherlands

Denhard De Smit

Netherlands
Sabine De Wall

Germany

Georgios Decavalas

Greece

Eugene Declercq

USA

Jocelyn Dejong

Lebanon

Anna Dencker

Sweden

Kebede Deribe

Ethiopia

Kristen Destigter

USA

Niveditha Devasenapathy

India

Nadia Diamond-Smith

USA

Jeffrey Dicke

USA

Paul Dielemans

Malawi

Anke Diemert

Germany

Lisabeth Dilalla

USA

Gary Dildy

USA

Jodie Dionne-Odom

USA

Jenna Dixon

Canada

Siobhan Dolan

USA

Vernon Dolinsky

Canada

Sara Donahue

USA

Amol Dongre

India

Jon Dorling

UK

Soo Downe

UK 
Danielle Downs

USA

Therese Dowswell

UK

Ann Dozier

USA

Alison Drake

USA

Rita Driggers

USA

Yunden Droma

Japan

Rohan D'Souza

Canada

Guillaume Ducarme

France

Kirsty Dundas

UK

Jill Durocher

USA

Johannes Duvekot

Netherlands

Els Duysburgh

Belgium

Susie Dzakpasu

Canada

Keith Eastwood

Australia

Joyce Edmonds

USA

Andrew Edmonds

USA

Anbrasi Edward

USA

Bolaji Egbewale

Nigeria

Torbjørn Moe Eggebø

Norway

Anne Eglash

USA

Anne Ego

France

Diane Ehlers

USA
Clara Ejembi

Nigeria

Tipaya Ekalaksananan

Thailand

Maria Ekelin

Sweden

Cecilia Ekéus

Sweden

Anette Ekström

Sweden

Alison El Ayadi

USA

Rajavel Elango

Canada

Mohamed El-Gharib

Egypt

Kirsty Elliott-Sale

UK

Khalifa Elmusharaf

Ireland

Khalifa Elmusharaf

Sudan

Ruth Endacott

UK

Daniel Enquobahrie

USA

Sonja Entringer

Germany

Yeetey Enuameh

Ghana

Offer Erez

Israel

Carlos Escudero

Chile

Anne Eskild

Norway

Birgitta Essén

Sweden

Michael Evangeli

UK

William Evans

USA

E Eworuke

USA
Marijke Faas

Netherlands

Pam Factor-Litvak

USA

Adeniyi Fagbamigbe

Nigeria

Titilayo Fakeye

Nigeria

Abiodun Fakokunde

UK

Mohamed Fathalla

Egypt

Ahbab Mohammad Fazle Rabbi

Bangladesh

Denice Feig

Canada

Marlena Fejzo

USA

Anis Feki

Switzerland

Helen Feltovich

USA

Tanis Fenton

Canada

Shingairai Feresu

South Africa

Shingairai Feresu

USA

Nina Ferrari

Germany

Zach Ferraro

Canada

Catherine Fetherston

Australia

Tamara Fetters

USA

Andrea Fielder

Australia

Kenneth Finlayson

UK

Tabassum Firoz

Canada

Jane Fisher

Australia 
Gerry Fitzgerald

Australia

Joel Fokom Domgue

Cameroon

Jane Ford

Australia

Della Forster

Australia

\section{Edward Fottrell}

UK

Maralyn Foureur

Australia

Karin Anneliese Fox

USA

Ana Beatriz Franco-Sena

Brazil

Lynn Freedman

USA

Atle Fretheim

Norway

Eleanor Friedman

USA

Robert Fryatt

South Africa

Matthew Fuller-Tyszkiewicz

Australia

Noya Galai

USA

Nicolas Galazis

UK

Colleen Gallagher

USA

Jenny Gamble

Australia

Hilary Gammill

USA

Rebecca Ganann

Canada

Robin Gandley

USA

Ashalatha Ganesh

India

John Kuumuori Ganle

Ghana
Jason Gardosi

UK

Michel Garenne

France

Ester Garne

Denmark

Lisa Garnweidner-Holme

Norway

David Garry

USA

Deirdre Gartland

Australia

Anca Gaston

Canada

Tania Gavidia

Australia

Abebaw Gebeyehu

Ethiopia

Alem Gebremariam

Ethiopia

Stacie Geller

USA

Compaoré Georges

Burkina Faso

Ioannis Germanakis

Greece

Melanie Gibson-Helm

Australia

Mark Giganti

USA

Christopher Gill

USA

Wendy Gilleard

Australia

Sylvie Girard

Canada

Carmen Giurgescu

USA

Norman Goco

USA

Sonu Goel

India

Laura Goetzl

USA
Rosa Gofin

USA

Robert Goldenberg

USA

Srinivas Goli

India

Bruna Gonçalves Cordeiro Da Silva Brazil

Adrienne Gordon

Australia

Dana Gossett

USA

Gillian Gould

Australia

Aimee Grant

UK

Holly Grason

USA

Michael Gravett

USA

James Greenberg

USA

Trisha Greenhalgh

UK

Karleen Gribble

Australia

Celia Grigg

Australia

Rosalie Grivell

Australia

Chad Grotegut

USA

Christian Gruber

Austria

Imma Guerras

Switzerland

Elvira Guerra-Shinohara

Brazil

Marie-Julia Guittier

Switzerland

Nalika Gunawardena

Spain

Erica Gunderson

USA 
Kemal Güngördük

Turkey

Juan De Dios Gutierrez Henares UK

Rengin Guzel

Turkey

Wilfried Gyselaers

Belgium

David Haas

USA

Dominic Haazen

USA

David Hackney

USA

Rosalind Haddrill

UK

Helen Haines

Australia

Nafisa Halim

USA

Arja Halkoaho

Finland

Wendy Hall

Canada

Jennifer Hall

USA

Michael Hambidge

USA

Patti Hamilton

USA

Karin Hammarberg

Australia

Shanshan Han

Australia

Anette Tarp Hansen

Denmark

Lotte Hardman

UK

Donna Hartz

Australia

Emily Harville

USA

Toshiyuki Hata

Japan
Abigail Hatcher

South Africa

Yvonne Hauck

Australia

Claudia Hawkins

USA

Marquis Hawkins

USA

Nicola Hawley

USA

Mark Hayter

UK

Honggu He

Singapore

Patricia Healy

Ireland

Maureen Heaman

Canada

Alexander Heazell

UK

Kelsey Hegarty

Australia

Michael Helewa

Canada

Rebecca Helmreich

USA

Julie Hennegan

UK

Amanda Henry

Australia

Erin Henshaw

USA

Monika Hermann

France

Berenice Herve

France

Nicola Heslehurst

UK

Melissa Hill

UK

Zelee Hill

UK

Janet Hirst

UK
Ha Hoang

Australia

Stephen Hodgins

USA

Matthew Hoffman

USA

Justus Hofmeyr

South Africa

Theresa Hoke

USA

Sarah Jane Holcombe

USA

Jennifer Hollowell

UK

Sara Holton

Australia

Malin Holzmann

Sweden

Caroline Homer

Australia

Lucy Hope

UK

Kristine Hopkins

USA

Dell Horey

Australia

Bernardo Horta

Brazil

Jaime Horton

USA

Tsu-Hsin Howe

USA

Carl Hubel

USA

Tai-Ho Hung

Taiwan

Billie Hunter

UK

Darren Hutchinson

Australia

Marianne Hutti

USA

Lieven Huybregts

Belgium 
Zubairu Iliyasu

UK

Por Ir

Cambodia

Jillian Ireland

UK

Farah Islam

Canada

Nazrul Islam

Canada

Ann-Charlotte Iversen

Norway

Jonathan Ives

UK

Milena Jacobs

Australia

B. Jager

Netherlands

Jennifer Jao

USA

Tara Jatlaoui

USA

Sangita Jindal

USA

Rosalind John

UK

Guro Mørk Johnsen

Norway

Sophia Johnson

Australia

Julie Jomeen

UK

Eleri Jones

UK

Catriona Jones

UK

K Joseph

Canada

Chandni Joshi

Australia

Andre Junqueira Caetano

Brazil

Alisa Kachikis

USA
Erkan Kalafat

Turkey

Yogavijayan Kandasamy

Australia

Lisa Kane Low

USA

Anil Kapur

Denmark

Anita Kar

India

Polyxeni Karakosta

Greece

Ali Karim

Ethiopia

Rajendra Karkee

Nepal

Bekir Karlik

Turkey

Spyridon Karras

Greece

Linda Kaste

USA

Jennifer Kastello

USA

Noriko Kato

Japan

Gilles Kayem

France

Abolanle Kayode

Nigeria

Xiayi Ke

UK

Bekana Kebede

Ethiopia

Bryn Kemp

UK

Garth Kendall

Australia

Holly Kennedy

USA

Remon Keriakos

UK

Sarah Finocchario Kessler USA
Erum Khan

UK

Michelle Khan

USA

Sunil Khanna

USA

Resham Khatri

Nepal

Yasser Khazaal

Switzerland

Chong Jai Kim

USA

Carol Kingdon

UK

Dawn Kingston

Canada

John Kinuthia

Kenya

Russell Kirby

USA

Kristen Kjerulff

USA

Michael Klein

Canada

Marie Klingberg-Allvin

Sweden

Marian Knight

UK

Theano Kokkinaki

Greece

Klaas Koop

Netherlands

Ounjai Kor-Anantakul

Thailand

Irene Korstjens

Netherlands

Kristine Koski

Canada

Christopher Kovacs

Canada

Zoltan Kozinszky

Sweden

Alexander Krafft

Switzerland 
Joan Kraft

USA

Ghattu V Krishnaveni

India

Bharati Kulkarni

India

Sailesh Kumar

Australia

Santosh Kumar

India

G Anil Kumar

India

Beenu Kushwah

India

Linda Kvist

Sweden

Barbara Kwast

Netherlands

Alain Labrique

USA

Robert Lachmann

Germany

Ching Tat Lai

Australia

Samantha Lain

Australia

Katariina Laine

Norway

Joan Lalor

Ireland

Yvonne Lamers

Canada

Ronald Lamont

UK

Michelle Lampl

USA

Stuart Lanham

UK

Sophia Lannon

USA

Hermann Lanou

Burkina Faso

Samia Laokri

USA
Malinee Laopaiboon

Thailand

Jacob Larkin

USA

Sarah Larkins

Australia

Elysia Larson

USA

Denise Lawler

Ireland

Beverley Lawton

New Zealand

Brian Layden

USA

Liana Leach

Australia

Nicky Leap

Australia

Charlotte Leboeuf-Yde

Denmark

Brigitte Leeners

Switzerland

Paul Leeson

UK

Kathryn Lemery-Chalfant

USA

Tiziana Leone

UK

Mayri Sagady Leslie

USA

Michael Levin

USA

Beth Lewis

USA

Zhiwen Li

China

Li Li

China

Changwei Li

USA

Linlin Li

USA

Ana Maria Linares

USA
Robert Liston

Canada

Helena Litorp

Sweden

Stephan Litschig

Spain

Tingting Liu

USA

Elisa Llurba

Spain

Yew L Lo

Singapore

Silvia Lobmaier

Germany

Robert Locke

USA

Ulrika Rehnström Loi

Sweden

Ladeisha Lombard

Kenya

Andres Lopez Bernal

UK

Jorge Lopez-Camelo

Argentina

Enrico Lopriore

Netherlands

Teresa Loureiro

Portugal

Julia Lowe

Canada

Mirjam Lukasse

Norway

Ingela Lundgren

Sweden

Riitta Luoto

Finland

Jacob Alexander Lykke

Denmark

Kimberly Ma

USA

Luis Machado Junior

Brazil

David Macintyre

UK 
Kevin Mackway-Jones

UK

Bvudzai Priscilla Magadzire

South Africa

Everett Magann

Per Magnus

Norway

Dean Maharaj

New Zealand

Smarajit Maiti

India

Eva Malacova

Australia

Maheen Malik

USA

Mats Malqvist

Sweden

Caroline Maltepe

Canada

Nataša Mandić

Slovenia

Gwendolin Manegold-Brauer

Switzerland

Bradley Manktelow

UK

Judith Mannien

Netherlands

Tanya J Marchant

UK

Colin Martin

UK

Pierre Martin-Hirsch

UK

Wellington Martins

Brazil

Katie Marvin-Dowle

United Kingdom

Vicki Masson

New Zealand

Jiji Mathews

India

Ilan Matok

Israel
Shigeki Matsubara

Japan

Shinya Matsuzaki

Japan

Anne Matthews

Ireland

Mandy Matthewson

Australia

Linda May

USA

Maureen Mayhew

Canada

Lawrence Mbuagbaw

Cameroon

Anthony Desmond Mccarthy

Argentina

Affette Mccaw-Binns

Jamaica

Christine Mccourt

UK

Judith Mccoyd

USA

Jean Mccrory

USA

Sheila Mcdonald

Canada

Alison Mcfadden

UK

Elizabeth Mcfarlane

USA

Rhona Mcinnes

UK

Meredith Mcintyre

Australia

Britt Mckinnon

Canada

Helen Mclachlan

Australia

Monica Mclemore

USA

Azar Mehrabadi

Canada

Yared Mekonnen

Ethiopia
Vn Melnikov

Russian Federation

Sara Meltzer

Canada

Ramkumar Menon

USA

Fiona Mensah

UK

Astrid Merkx

Netherlands

Jill Mhyre

USA

Ellen Mikkelsen

Denmark

Anne Mills

UK

Lesley Milne

UK

Abul Milton

Australia

Maria Amelia Miquelutti

Brazil

Adriana Miranda-Ribeiro

Brazil

Fadi Mirza

Lebanon

Dawn Misra

USA

Blandina Theophil Mmbaga

Norway

Markus Mohaupt

Switzerland

Rob Mooij

Netherlands

Lwendo Moonzwe Davis

USA

Thomas Moore

USA

José Morales-Roselló

Spain

Kate Morgaine

UK

Alison Morgan

Australia 
Emmanuel Morhe

Ghana

Naho Morisaki

Japan

David Morris

UK

Shaine Morris

USA

Leonard Morssink

Netherlands

Andrea Mosher
Canada

Cheryl Moyer

USA

Rose Mpembeni

Tanzania

Mwifadhi Mrisho

Tanzania

Kathryn Muldoon

Ireland

Shama Munim

Pakistan

Vanessa Murphy

Australia

Sylvia Murphy Tighe

Ireland

Philippa Musoke

Uganda

Leslie Myatt

USA

Judith Myers

Australia

Jenny Myers

UK

Shamsun Nahar

Saudi Arabia

Manisha Nair

UK

Jane Nakawesi

Uganda

Katsuhiko Naruse

Japan

Mzikazi Nduna

South Africa
Ellen Nelissen

Tanzania

Britt-Ingjerd Nesheim

Norway

Charlotte Neumann

USA

James Newham

UK

Sam Newton

Ghana

Judith Nguimfack

Senegal

Michael Nicholl

Australia

Marianne Nieuwenhuijze

Netherlands

Anna Nikonova

Somashekhar Nimbalkar

India

Tanya Nippita

Australia

Veeranoot Nissapatorn

Malaysia

Aggie Noah

USA

Karen Noblett

USA

Joy Noel-Weiss

Canada

Barbara Nolens

Uganda

Marcelo Luís Nomura

Brazil

Judith Noronha

India

Kathleen Norr

USA

Uchenna Nwagha

Nigeria

Angelo Nyamtema

Tanzania

Laura Oakley

UK
Andrew Obi

Nigeria

Beverley O'Brien

Canada

Pat O'Brien

UK

Louise O'Brien

USA

John O'Brien

USA

Dianne O'Connell

Australia

Thomas O'Connell

USA

Willie Oglesby

USA

Eric Ohuma

UK

Ugochukwu Vincent Okafor

Nigeria

Maureen Okam

USA

John Okanda

Kenya

Niran Okewole

Nigeria

Kehinde Okunade

Nigeria

Ellinor Olander

UK

Joann O'Leary

USA

Per Olofsson

Sweden

Beth Olson

USA

Bolajoko O. Olusanya

Nigeria

Siti Zawiah Omar

Malaysia

Sinead M. O'Neill

Ireland

Sam Ononge

Uganda 
Dorothy Ononokpono

Nigeria

Uchenna Onwudiegwu

Nigeria

Azubuike Onyebuchi

Nigeria

Arwa Ooweis

Jordan

Baafuor Opoku

Ghana

Waseem Osman

UK

Koyejo Oyerinde

USA

Agnieszka Pac

Poland

Ana Palei

USA

Julie Pallant

Australia

Shanta Pandey

USA

Mary Panjari

Australia

Min Hae Park

UK

Samantha Parker

USA

Leslie Parker

USA

Crystal Patil

USA

Thelma Patrick

USA

Robert Clive Pattinson

South Africa

Beth Payne

Canada

Sallie Pearson

Australia

Christopher Pell

Netherlands

Chun Peng

Canada
Gavin Pereira

USA

Patricia Perrenoud

Switzerland

Eva Persson

Sweden

Margareta Persson

Sweden

Gitte Lindved Petersen

Denmark

Manuela Pfinder

Germany

Pamela Pilkington

Australia

M Halit Pinar

USA

Catherine Pirkle

USA

Angela Poat

UK

Jashvant Poeran

USA

Wendy Pollock

Australia

Albrecht Popp

Switzerland

Joseph Potter

USA

Theresa Powell

USA

Robert Powers

USA

Kathleen Powis

USA

Rina Pradhan

Australia

Ndola Prata

USA

Busadee Pratumvinit

Thailand

Walter Prendiville

France

Dolores Pretorius

USA
Holly Priddis

Australia

Colin Pritchard

UK

Victor Pulgar

USA

Shuby Puthussery

UK

Qibin Qi

USA

\section{Chunfang Qiu}

USA

Edward Quadros

USA

Bernardo Queiroz

Brazil

Ingela Radestad

Sweden

Sari Räisänen

Finland

Augustine Rajakumar

USA

Shanti Raman

Australia

Louise Randall

Australia

Gopi Rangan

Australia

Jean Rankin

UK

Kristen Rappazzo

USA

Salah Rasheed

Egypt

Svein Rasmussen

Norway

Margaret Rayman

UK

Juliet Rayment

UK

Camille Raynes-Greenow

Australia

Peter Rebeiro

USA 
Maggie Redshaw

UK

\section{Annette Regan}

Australia

Donna Rennie

Canada

Decio Ribeiro Sarmento

Timor-Leste

Machteld Rijken

Netherlands

Marcus Rijken

Netherlands

Marcus Rijken

Thailand

Marlies Rijnders

Netherlands

Claire Roberts

Australia

Scott Roberts

USA

Michael Robson

Ireland

Lucia Rocca

UK

Corinne Rocca

USA

Lucia Rocca-Ihenacho

UK

Anna Joy Rogers

USA

Michael Ross

USA

Cristina Rossi

Italy

Heather Rowe

Australia

Rachel Rowe

UK

Sam Rowlands

UK

Alison Roxby

USA

Irene Ruengkhachorn

Thailand
Stephen Rulisa

Rwanda

Alice Rumbold

Australia

Rachel Rundle

UK

Mahama Saaka

Ghana

Lora Sabin

USA

Jayaprakash Sahoo

India

Yoshifumi Saisho

Japan

Carol Sakala

USA

Mihretab Melesse Salasibew

UK

Sarah Saleem

Pakistan

Jason Salemi

USA

Kjell Salvesen

Norway

Silvia Salvi

Italy

Juan Sanchez-Esteban

USA

Amarpreet Sandhu

USA

Valeria Sandrim

Brazil

Harshadkumar Sanghvi

USA

Ayesha Sania

USA

Gillian Santorelli

UK

Benn Sartorius

South Africa

Appunni Sathiya Susuman

South Africa

Felix Sayinzoga

Rwanda
Angela Scheuerle

USA

Crystal Schiller

USA

Virginia Schmied

Australia

Christentze Schmiegelow

Denmark

Francisco Schneuer

Australia

Ewoud Schuit

Netherlands

Hilary Schwandt

USA

Christiane Schwarz

Germany

Jane Scott

Australia

James Scott

USA

Nancy Scott

USA

Kerem Doga Seckin

Turkey

Olutoyin Sekoni

Nigeria

Daniel Sellen

Canada

Katherine Semrau

USA

Marie Seraphin

USA

Magdalena Serpa

USA

Alberto Severini

Canada

Haleema Shakur

UK

Donat Shamba

Tanzania

Antonia Shand

Australia

Sudesh Raj Sharma

Nepal 
Sheetal Sharma

UK

Kathryn Sharma

USA

Abigail Sharpe

UK

Phyllis Sharps

USA

Jill Shawe

UK

Sherif Shazly

Egypt

Kayleigh Sheen

UK

Zoe Sheppard

UK

Jill Sherriff

Australia

Eiji Shibata

Japan

Solomon Shiferaw

Ethiopia

Mary Shilalukey Ngoma

Zambia

Brian Shine

UK

Shashikant Sholapurkar

UK

Lauren Shomaker

USA

Binjwala Shrestha

Nepal

Dimitrios Siassakos

UK

Estelle Monique Sidze

Kenya

Leigh Signal

New Zealand

Robert Silver

USA

Kari Simonsen

USA

Marlene Sinclair

UK
Deepti Singh

India

Kavita Singh

USA

Heather Sipsma

USA

Pauline Slade

UK

Nancy L Sloan

USA

Jennifer Slyker

USA

Marrit Smit

Netherlands

Sian Smith

Australia

Valerie Smith

Ireland

Debbie Smith

UK

Jeffrey Michael Smith

USA

Laura Sockol
USA

Malin Soederberg

Sweden

Leslie Sokolow

USA

Nancy Soliman

Canada

Andrea Solnes Miltenburg

Tanzania

Priya Soma-Pillay

South Africa

Sajid Soofi

Pakistan

Alexandros Sotiriadis

UK

David Southall

UK

Jonathan Spector

USA

Rachael Spencer

UK
Juliane Spiegler

Germany

Jiri Spilka

Czech Republic

Chandrashekhar Sreeramareddy

Nepal

Tomasina Stacey

UK

Anthony Staines

Ireland

Aleksandra Staneva

Australia

Joanna Stanley

New Zealand

Mona Stedenfeldt

Norway

Amie Steel

Australia

Phyllis Stein

USA

Gabrielle Stevens

USA

Joanna Stewart

New Zealand

Mary Stewart

UK

Elisa Stivanello

Italy

Kathrin Stoll

Germany

William Stones

UK

Gro Leite Størvold

Norway

William Story

USA

Patrick Stover

USA

Eric Strachan

USA

Katrine Strandberg-Laren

Denmark

Donna Strobino

USA 
Christopher Sudfeld

USA

Colin Sullivan

Australia

Bo Sun

China

Fernanda G C Surita

Brazil

Fernanda Surita

Brazil

Rosnah Sutan

Malaysia

Muslim Abbas Syed

Pakistan

Orsolya Szenczi

Hungary

Angela Taft

Australia

Harry Tagbor

Ghana

Ajay Talati

USA

Hana Tamim

Canada

Peng Chiong Tan

Malaysia

Geok Chin Tan

Malaysia

Li Tang

Australia

Jennifer Tang

Malawi

Hannah Tappis

USA

Marie Tarrant

Hong Kong

Nick Tatonetti

USA

Paula Tavrow

USA

Susan Tawia

Australia

Lee Taylor

Australia
Kate Teela

Canada

Mathurin Tejiokem

Cameroon

Daniel Terry

Australia

Megan Teychenne

Australia

Zaneta Thayer

USA

Sudhin Thayyil

UK

Basky Thilaganathan

UK

Diana Thomas

USA

Susan Thompson

UK

Gill Thomson

UK

Ann Thomson

UK

Jim Thornton

UK

Andrea Tinelli

Italy

Christiana Titaley

Indonesia

William Wk To

Hong Kong

Angela Todd

Australia

Kathleen Tomsin

Belgium

Jocelyn Toohill

Australia

Maria Regina Torloni

Brazil

Siranda Torvaldsen

Australia

Sally Tracy

Australia

Naomi Trengove

Australia
Elizabeth Triche

USA

Helen Trottier

Canada

Jone Trovik

Norway

Lawrence Tsen

USA

Janet Turan

USA

Amy Turitz

USA

Deborah Turnbull

Australia

Marcel Twickler

Belgium

Zia Ul-Haq

UK

Priti Upadhyay

Nepal

Marcelo Urquia

Canada

Michele Usuelli

Italy

Bettina Utz

Belgium

Felipe Vadillo-Ortega

Mexico

Zvi Vaknin

Israel

Mike Van Den Hof

Canada

Gitsels Van Der Wal

Netherlands

Linde Van Genugten

Netherlands

Evelien Van Limbeek-Reinaerts

Netherlands

An-Sofie Van Parys

Belgium

Jos Van Roosmalen

Netherlands

Lenie Van Rossem

Netherlands 
Julie Van Schalkwyk

Canada

Henk Van Stel

Netherlands

Giel Van Stralen

Netherlands

Edwin Van Teijlingen

UK

Christiaan Van Woerden

Netherlands

Jeroen Vanderhoeven

USA

Eszter Vanky

Norway

Peter Varga

Hungary

Jojy Varghese

Australia

Beena Varghese

India

Karolyn Vaughan

Australia

Lara Vaz

USA

Saraswathi Vedam

Canada

András Vereckei

Hungary

Corine Verhoeven

Netherlands

Georgia Verropoulou

Greece

Marilza Vieira Cunha Rudge

Brazil

Jenny Vieveen

Netherlands

Reeta Vijayaselvi

India

Dimuthu Vinayagam

UK

Angela Vinturache

Canada

Frauke Von Versen-Höynck

Germany
Kranti Vora

USA

Susan Walker

Australia

Lorraine Walker

USA

Colin Walsh

Australia

Denis Walsh

UK

Susan Walsh

USA

Yan-Ling Wang

China

Suqing Wang

China

Jane Warland

Australia

Charlotte Warren

Kenya

Susan Watt

Canada

Andrew Weeks

UK

Karen Weidert

USA

Adi Weintraub

Israel

Amanda Wendt

Germany

Benedict Weobong

Ghana

Mark Wetherell

UK

Robert White

USA

Clare Whitehead

Australia

Heather Whitford

UK

Melissa Whitworth

UK

Mariana Widmer

Switzerland
Therese Wiegers

Netherlands

Ayona Wijemanne

UK

Scott Wilkes

UK

Jeffrey Wilkinson

USA

Jane Willcox

Australia

Suzanne Willey

Australia

Melissa Wilson

USA

Ronee Wilson

USA

Barbara Wilson-Clay

USA

Christopher Winfree

USA

Pattanee Winichagoon

Thailand

Anke Witteveen

Netherlands

Donna Wixted

UK

Claire Wood

UK

Gang Wu

Netherlands

Pamela Xaverius

USA

Jose-Miguel Yamal

USA

Youli Yao

Canada

O. Yaw Addo

USA

Kojo Yeboah-Antwi

USA

Hung-Wen Yeh

USA

Jane Yelland

Australia 
Jeanine Young

Australia

Sera Young

USA

Juping Yu

UK
Antonella Zambon

Italy

Gerald Zavorsky

USA

Phyllis Zelkowitz

Canada
Yujia Zhang

United States

Qiu-Yue Zhong

USA

Patti Zuzelo

USA 